
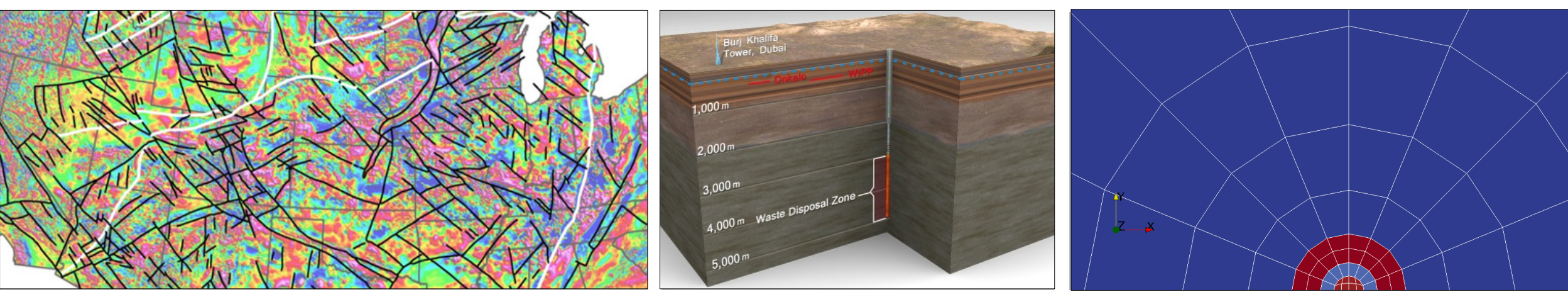

\title{
Site Characterization for the Deep Borehole Field Test
}

Kristopher L. Kuhlman

Sandia National Laboratories 


\section{What is the Deep Borehole Field Test?}

What are we trying to observe?

How are we planning on measuring it?

How is this field test unique? 


\section{Deep Borehole}

\section{- Disposal Concept}

- Possible robust isolation from shallow geosphere

- Barriers

- Depth

- Salinity \& perm. gradients

- Lack of driving forces

- Diffusion dominated

- Field Test

- $8.5^{\prime \prime} \& 17$ " boreholes to $5 \mathrm{~km}$

- Technical demonstration

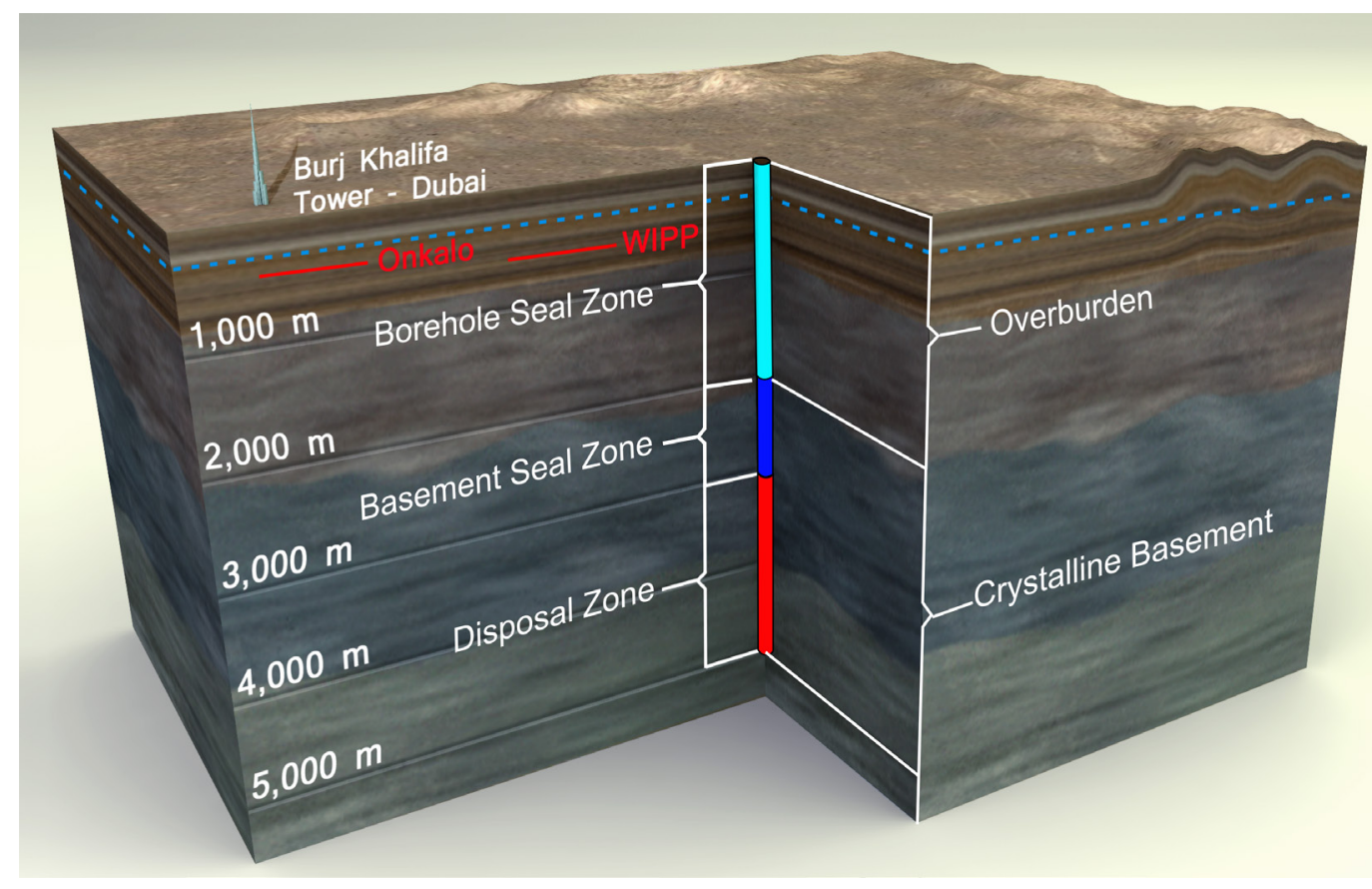

- Drilling

- Sampling \& in situ testing

- Surface/downhole handling

- No waste 


\section{Deep Borehole Conceptual Profiles}

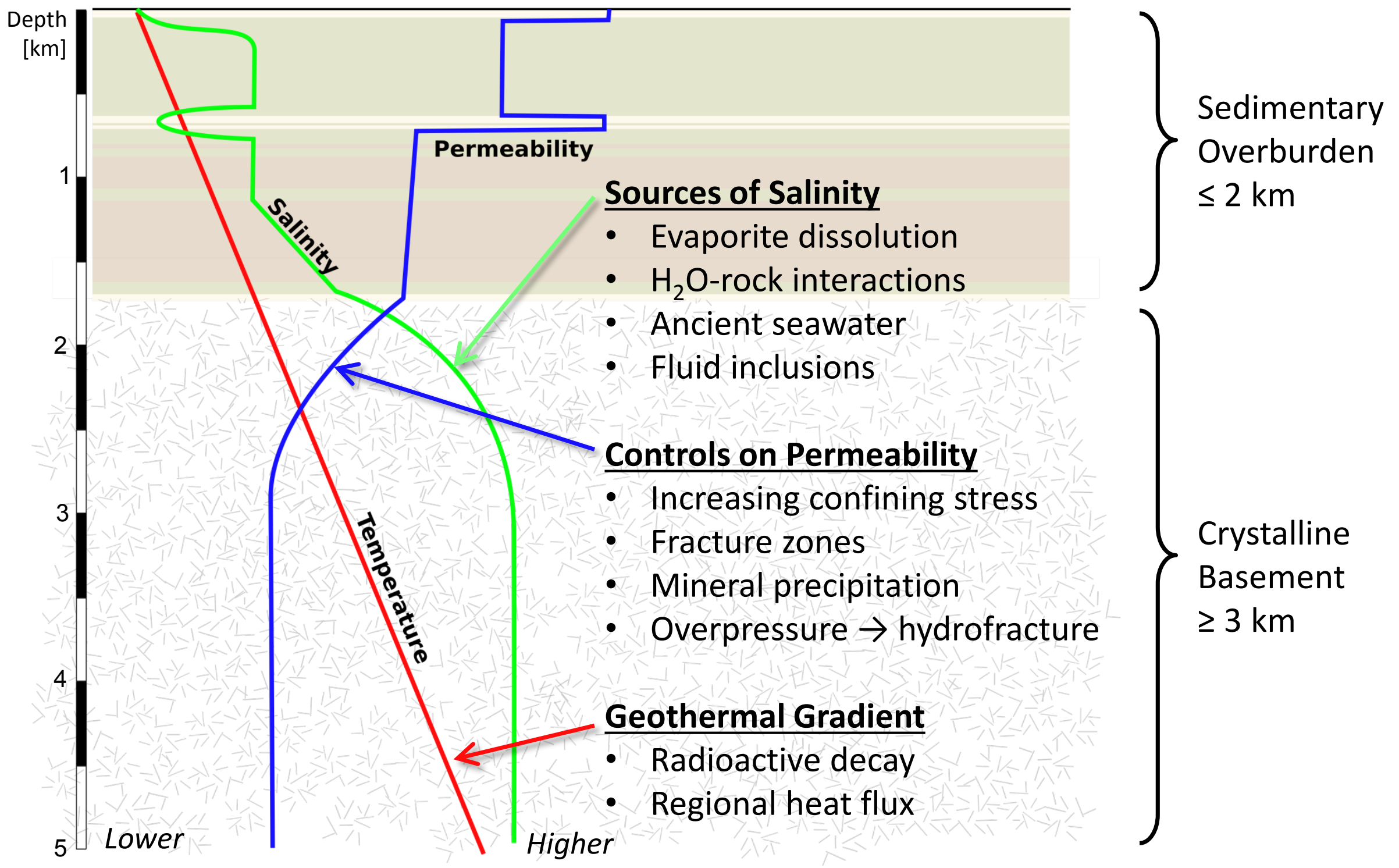




\section{Observed Profiles}

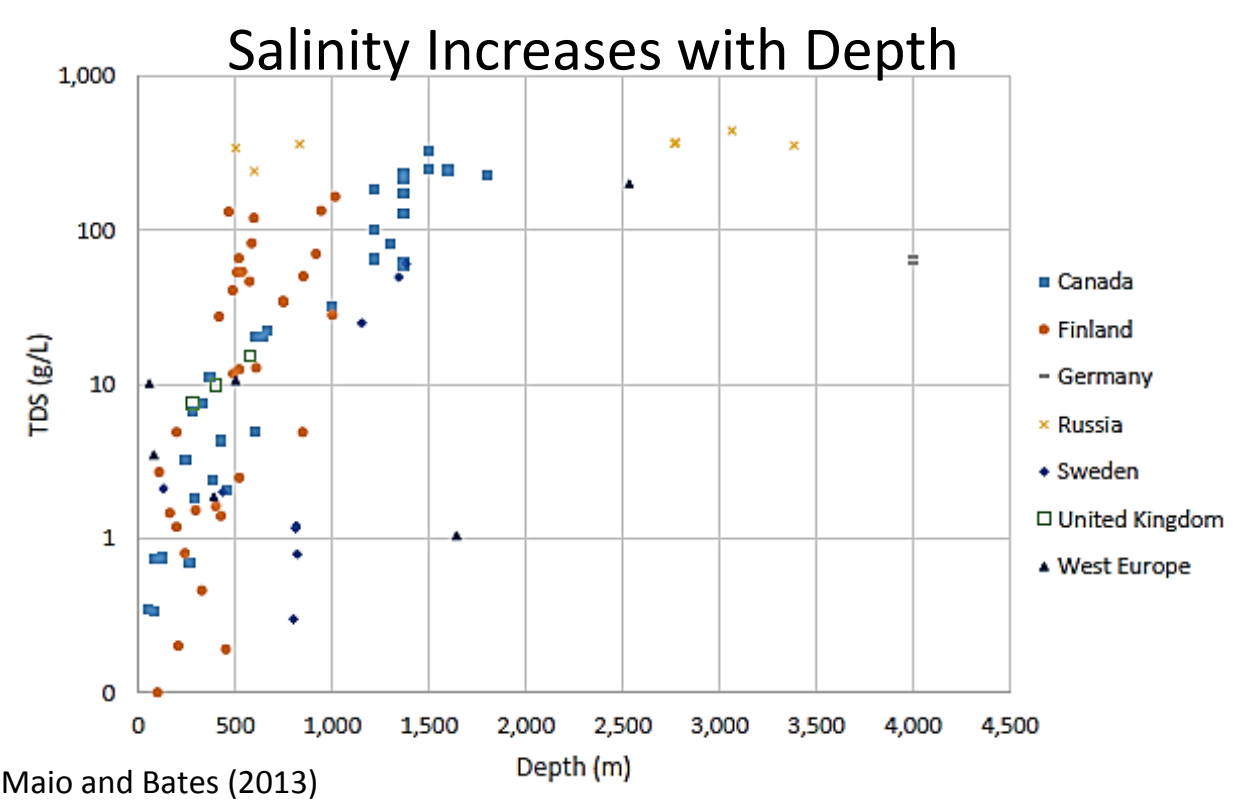

\section{Bulk Permeability Decreases with Depth}

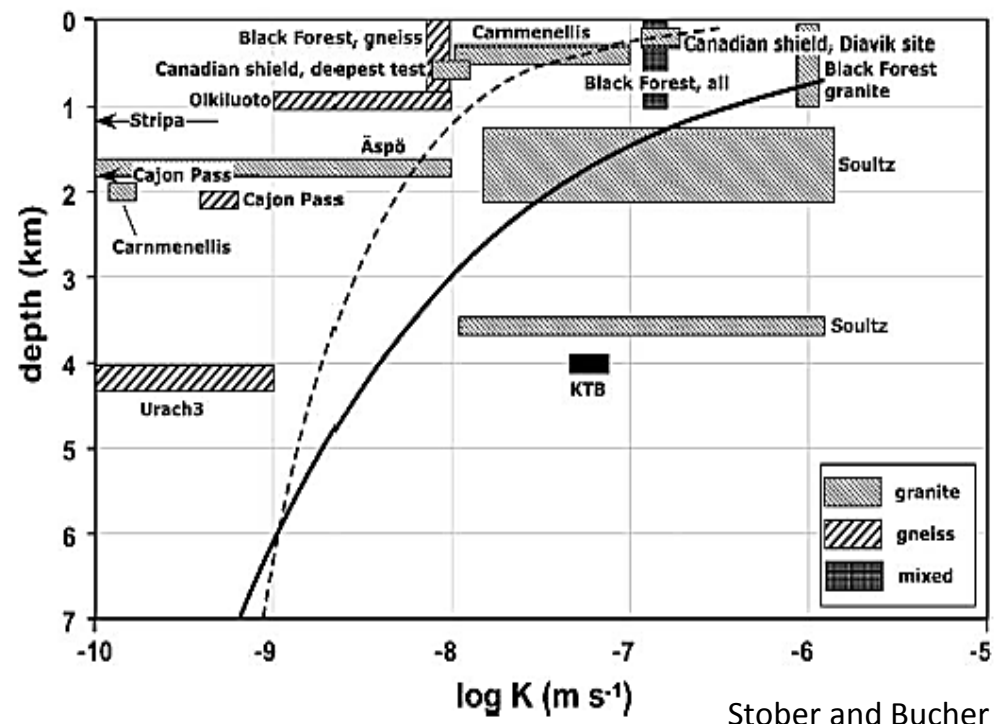

Stober and Bucher (2007) 


\section{Observed Profiles}

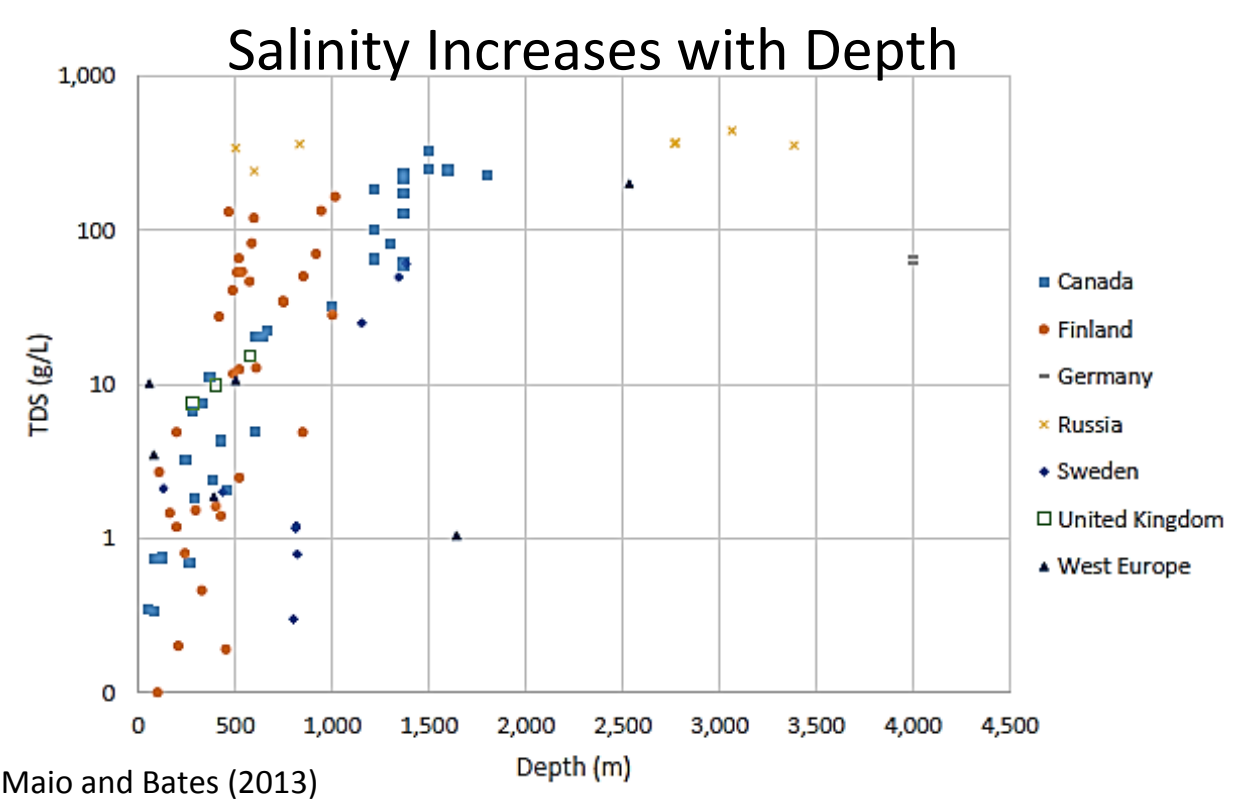

DeMaio and Bates (2013)

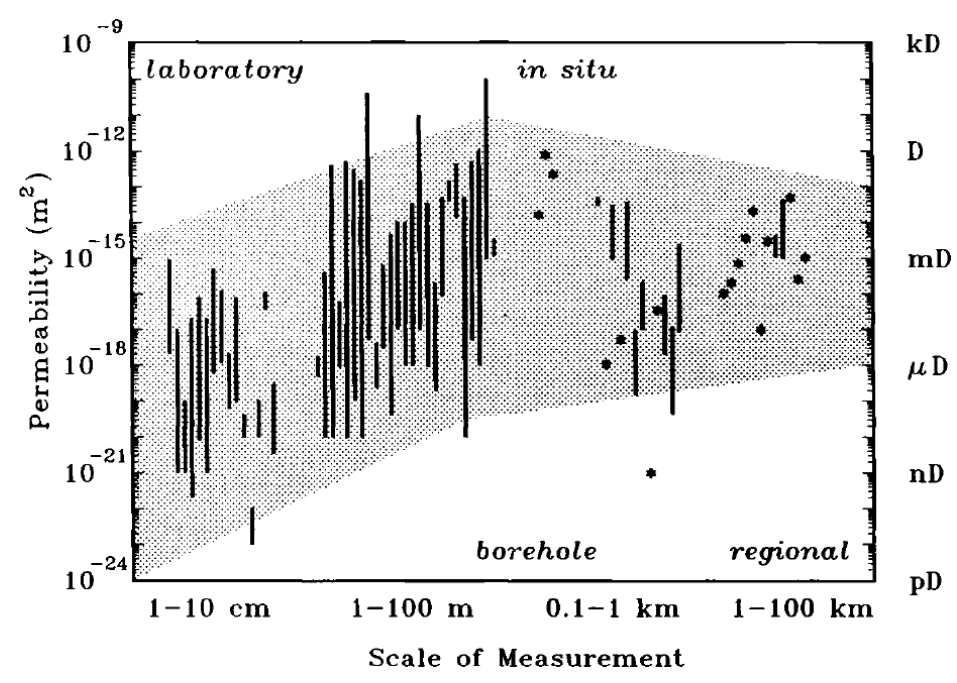

\section{Bulk Permeability Decreases with Depth}

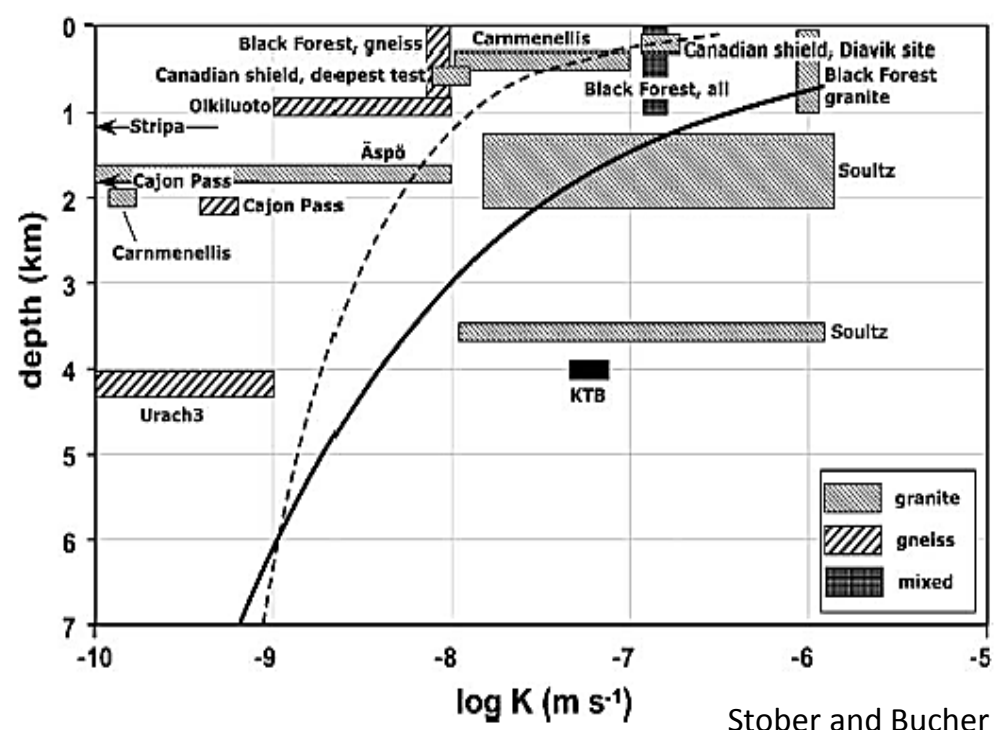

Stober and Bucher (2007)

\section{Bulk Permeability Increases with Scale Clauser (1992)}




\section{Observed Profiles}

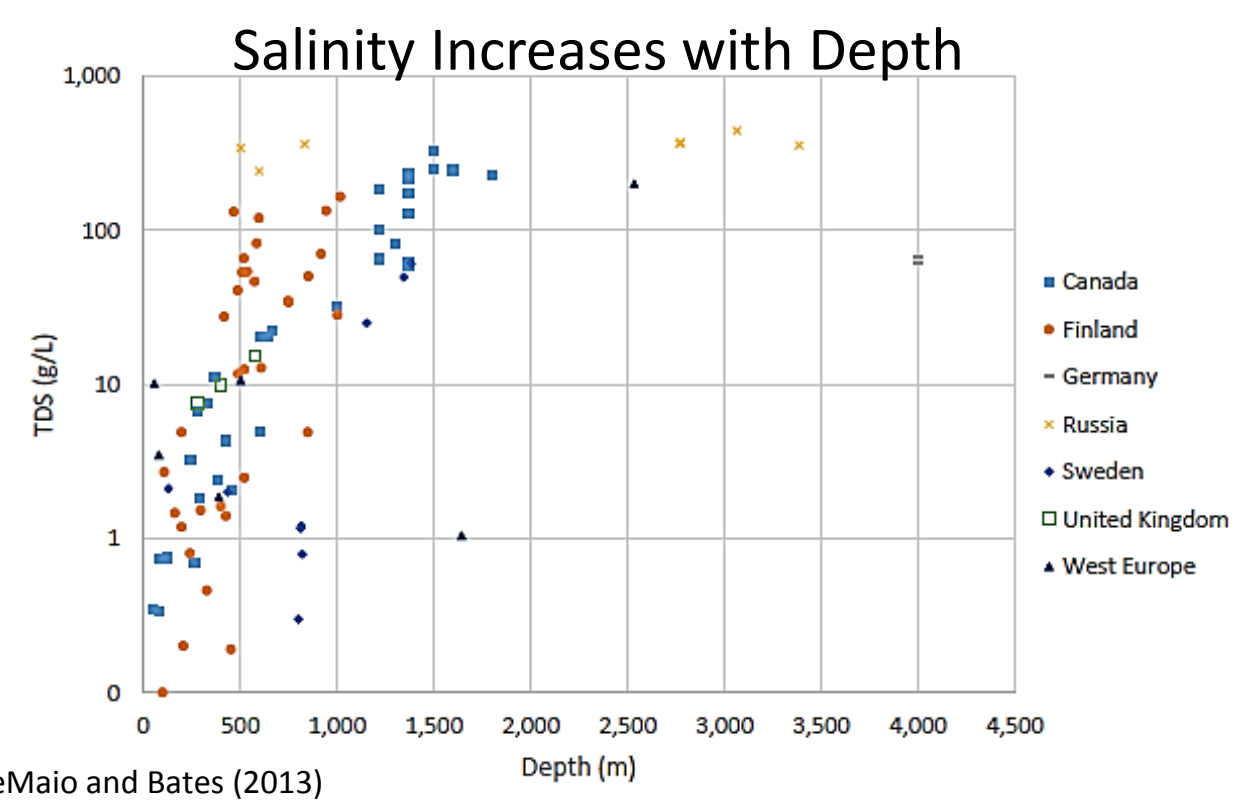

DeMaio and Bates (2013)

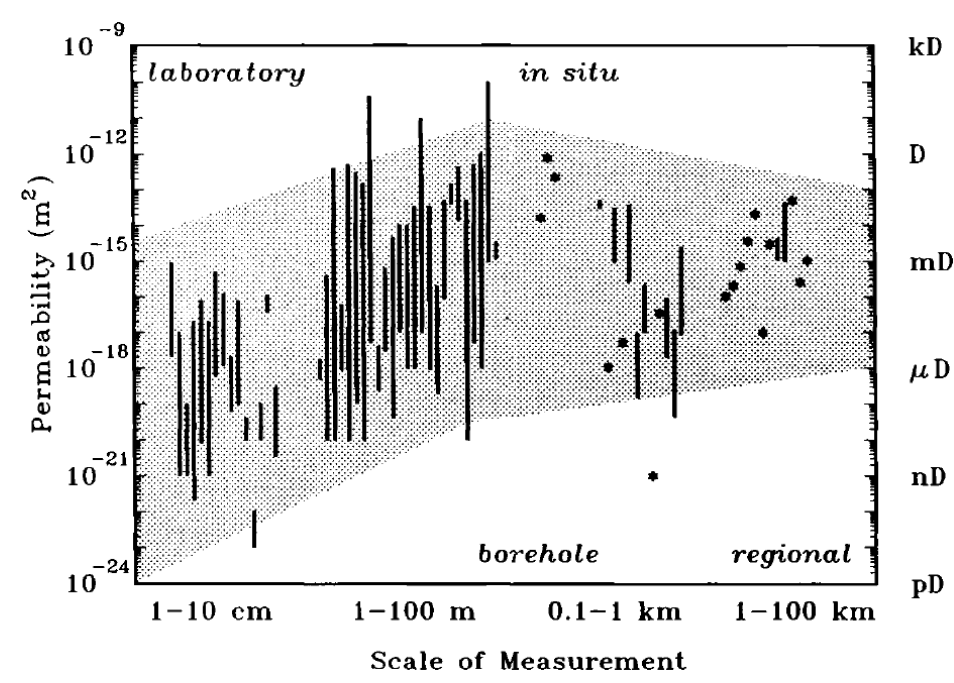

Bulk Permeability Decreases with Depth

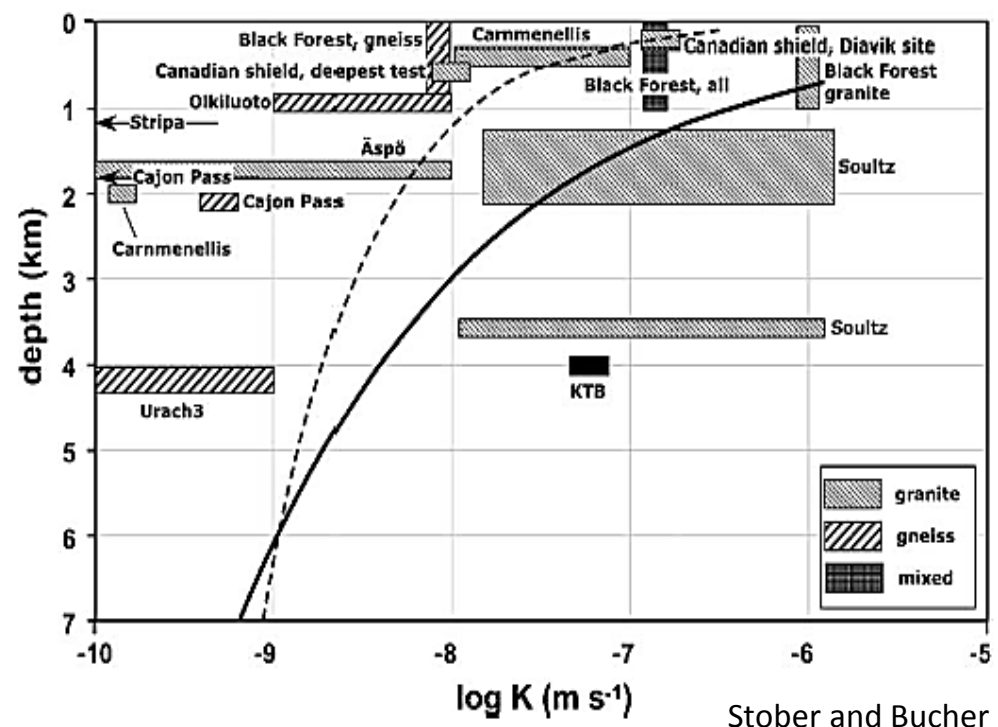

Stober and Bucher (2007)

Bulk Permeability Increases with Scale

Clauser (1992)

\section{Upscaling permeability data}

vs.

Geochemical composition and natural tracers data 


\section{Sampling Profiles}

- Borehole Geophysics

- Logging During Drilling

- Mud fluids/tracers/dissolved gases

- Basement Rock Samples

- Coring (5\%, $150 \mathrm{~m}$ total)

- Drill Cuttings/Rock Flour (XRD + XRF)

- Formation Fluid Samples

- Pumped from high-perm intervals

- Extracted from cores

- Formation Fluid (\& Mud) Analytes

- Onsite fluid density/temperature

- Major ions \& trace metals

- C, N, S, Sr \& U isotope ratios

- ${ }^{4}$ He buildup in fluids \& qtz. crystals

- Stable water isotopes

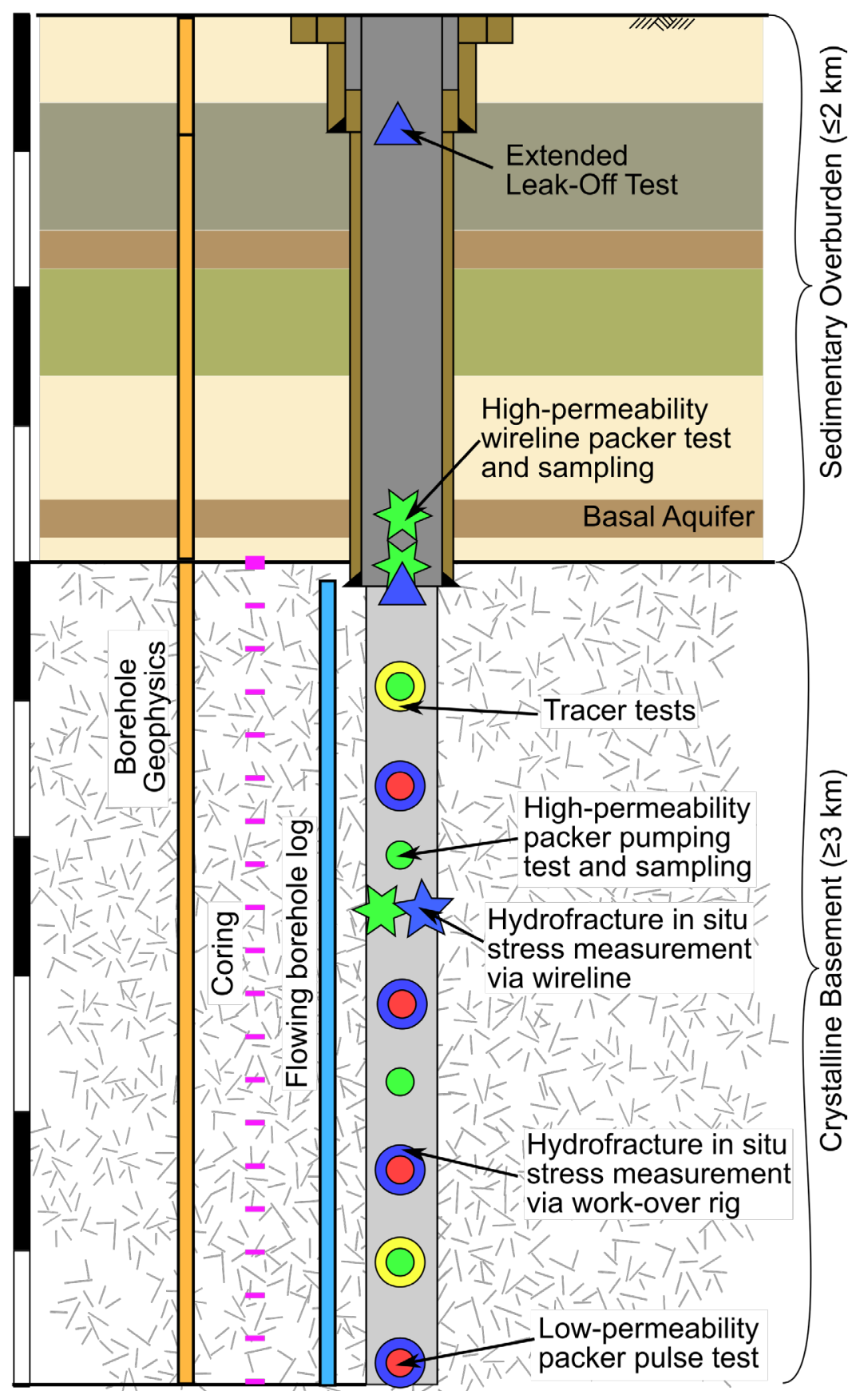




\section{In Situ Testing}

- Flowing Borehole Logs

- Salinity dilution \& temperature diffusion

- Hydrologic Tests

- Low-perm pulse tests (5)

- High-perm pumping tests (5)

- Estimate:

- Static formation pressure

- Permeability / compressibility / skin

- Injection-Withdrawal Tracer Tests (2)

- Hydromechanical Packer Test (1)

- Estimate $k_{\mathrm{DRZ}}(\sigma)$

- Hydraulic Fracturing Stress Tests (5)

- Estimate $\sigma_{\mathrm{h}} \& \sigma_{\mathrm{H}}$ magnitudes

- Test unfractured \& existing fracture intervals

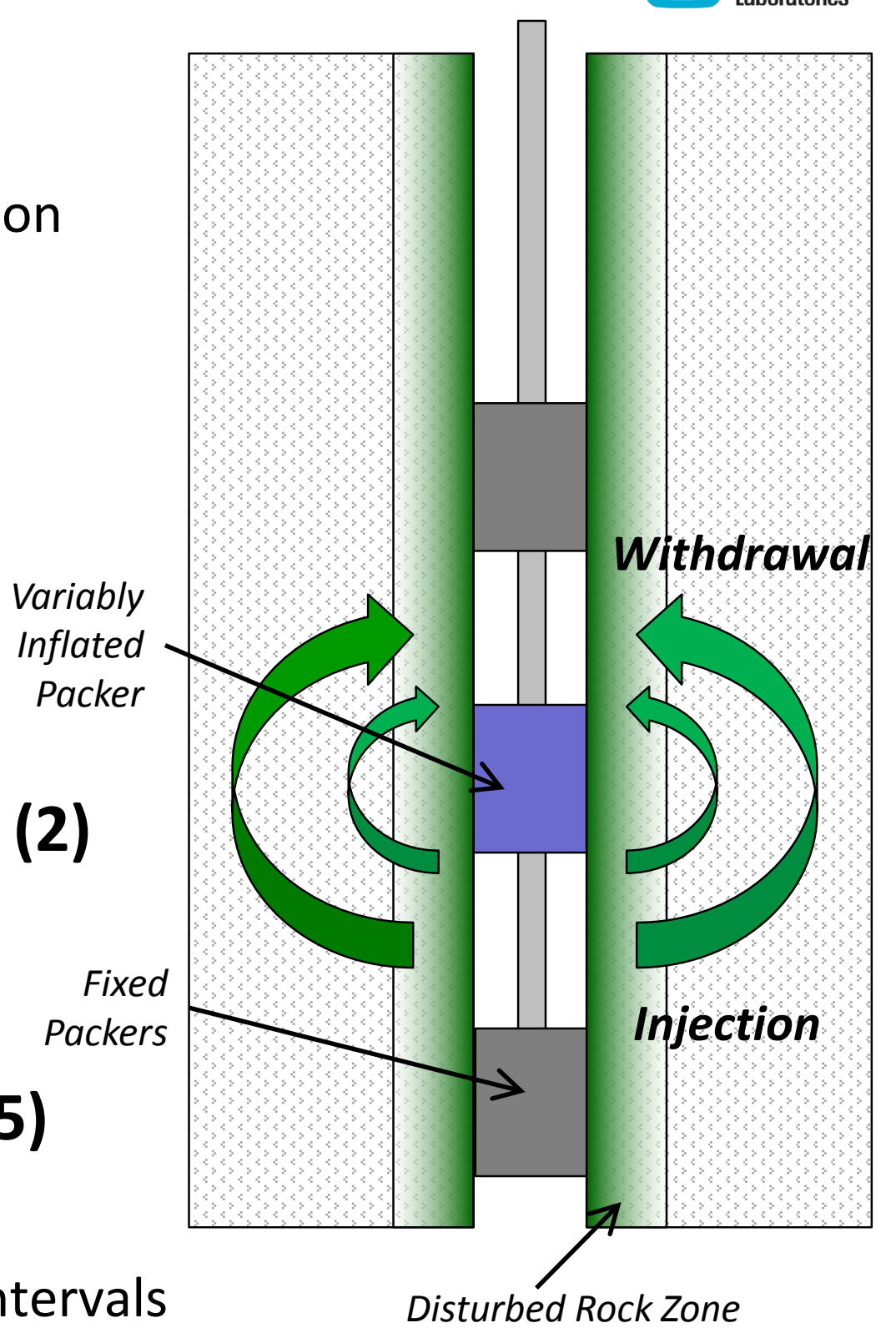




\section{Summary and Uniqueness}

- DBFT Likely Different From:

- Oil/gas or mineral exploration

(low perm., low porosity rocks)

- Geothermal exploration (low geothermal gradient)

- Shallow drilling/testing (high $p$, high $\sigma$, deep, breakouts)

- DBFT Characterization Approach

- Not exhaustive permeability characterization

- Seeking geochemical evidence of system isolation

- Use "off-the-shelf" approaches when available

- DBFT Goals

- Drill straight large-diameter boreholes to $5 \mathrm{~km}$ depth

- Demonstrate sample collection (cores + formation fluid)

- Enough samples

- Low enough contamination level

- Demonstrate in situ testing at depth (3 to $5 \mathrm{~km}$ ) 